\title{
Syllable and language model based features for detecting non-scorable tests in spoken language proficiency assessment applications
}

\author{
Angeliki Metallinou, Jian Cheng \\ Knowledge Technologies, Pearson \\ 4040 Campbell Ave., Menlo Park, California 94025, USA \\ angeliki.metallinou@pearson.com jian.cheng@pearson.com
}

\begin{abstract}
This work introduces new methods for detecting non-scorable tests, i.e., tests that cannot be accurately scored automatically, in educational applications of spoken language proficiency assessment. Those include cases of unreliable automatic speech recognition (ASR), often because of noisy, off-topic, foreign or unintelligible speech. We examine features that estimate signalderived syllable information and compare it with ASR results in order to detect responses with problematic recognition. Further, we explore the usefulness of language model based features, both for language models that are highly constrained to the spoken task, and for task independent phoneme language models. We validate our methods on a challenging dataset of young English language learners (ELLs) interacting with an automatic spoken assessment system. Our proposed methods achieve comparable performance compared to existing non-scorable detection approaches, and lead to a $21 \%$ relative performance increase when combined with existing approaches.
\end{abstract}

\section{Introduction}

Automatic language assessment systems are becoming a valuable tool in education, and provide efficient and consistent student assessment that can complement teacher assessment. Recently, there has been a great increase of English Language Learners (ELLs) in US education (Pearson, 2006). ELLs are students coming from nonEnglish speaking backgrounds, and often require additional teacher attention. Thus, assessing ELL student language proficiency is a key issue.

Pearson has developed an automatic spoken assessment system for K-12 students and collected a large dataset of ELL students interacting with the system. This is a challenging dataset, containing accented speech and speech from young students. Thus, for a small percentage of tests, it is technically challenging to compute an accurate automatic score, often because of background/line noise, off-topic or non-English responses or unintelligible speech. Such tests as referred to as non-scorable. Here, our goal is to propose new methods for better classifying non-scorable tests and describe a system for non-scorable detection.

We propose two new sets of features: syllable based and language model (LM) based. The intuition is to contrast information from different sources when processing a test, in order to detect inconsistencies in automatic speech recognition (ASR), that often appear in non-scorable tests. Syllable features measure similarity between different estimates of syllable locations, one extracted from ASR and the second from the raw signal. LM features measure similarity between two ASR results, one using a standard item specific word LM, and the second using a item independent phoneme LM. Finally, an additional set of ASR confidence scores and log-likelihoods is computed using the proposed phoneme LM.

Compared to existing work, our new methods achieve comparable performance, although they approach the problem from a different perspective. Furthermore, our proposed features carry complementary information to existing ones, and lead to a $21 \%$ relative performance increase when combined with existing work.

\section{Related Work}

A review of spoken language technologies for education can be found in Eskanazi (2009). There is a considerable amount of previous work on automatic speech assessment. Pearson's automated speech scoring technologies that measure the candidates' speaking skill (pronunciation, flu- 
ency, content) have been used in the Versant series tests: English, Aviation English, Junior English, Spanish, Arabic, French, Dutch, Chinese (Bernstein et al., 2000; Bernstein and Cheng, 2007; Cheng et al., 2009; Bernstein et al., 2010; Xu et al., 2012), and Pearson Test of English Academic (Pearson, 2011). A non-scorable detection component (Cheng and Shen, 2011) is usually required for such systems. Educational Testing Service described a three-stage system on spoken language proficiency scoring, that rates open-ended speech and includes a non-scorable detection component (Higgins et al., 2011).

The system described here evaluates spoken English skills of ELL students in manner and content. Past work on children's automatic assessment of oral reading fluency includes systems that score performance at the passage-level (Cheng and Shen, 2010; Downey et al., 2011) or word-level (Tepperman et al., 2007).

Regarding detecting problematic responses in speech assessment applications, related work includes off-topic and non-scorable detection. Nonscorable detection is a more general problem which includes not only off-topic responses, but also noisy, poor quality, foreign or unintelligible responses, etc. Higgins et al. (2011) describe a system that uses linear regression and four informative features (number of distinct words, average ASR confidence, average and standard deviation of speech energy) for filtering out non-scorable responses. Yoon et al. (2011) use a set of 42 signalderived and ASR features along with a decision tree classifier for non-scorable response detection. Many of their features are also extracted here for comparison purposes (see Section 7).

Chen and Mostow (2011) focus on off-topic detection for a reading tutor application. They use signal features (energy, spectrum, cepstrum and voice quality features) and ASR features (percentage of off-topic words) with a Support Vector Machine (SVM) classifier. In our previous work (Cheng and Shen, 2011), we described an off-topic detection system, where we computed three variations for ASR confidence scores, along with features derived from acoustic likelihood, language model likelihood, and garbage modeling. Linear regression was used for classification.

Here, we focus on non-scorable test detection, using aggregate information from multiple test responses. We propose new similarity features that are derived from syllable location estimation and the use of a item independent phoneme LM.

\section{The ELL student dataset}

\subsection{The asessment system}

Pearson has developed an English proficiency assessment test, which has been administered in a large number of K-12 ELL students in a U.S. state. The speaking component of the test is delivered via speakerphone, and the student performance is automatically scored. Each tests consists of a series of spoken tasks which are developed by professional educators to elicit various displays of speaking ability. There are repeat tasks, where students repeat a short sentence, and open ended tasks, where students are required to answer questions about an image or a topic, give instructions, ask a question about an image, etc. Each test contains multiple test prompts (also referred to as items), some of which may belong to the same task. For example, for the 'question about image' task, there may be items refering to different images. Each test contains student responses to the items. Responses which are typically two or three sentences long.

Figure 1 summarizes the components of Pearson's automatic proficiency assessment system. Assessment is done through combination of ASR, speech and text processing, and machine learning to capture the linguistic content, pronunciation and fluency of the student's responses. In this work, we focus on the lower block of Figure 1 that illustrates the non-scorable detection component, whose purpose is to detect the tests that cannot be reliably scored. It exploits signal related and ASR information to extract features that are later used by a binary classifier to decide whether a test is scorable or not. Our goal is to filter out nonscorable tests, to be graded by humans. The proficiency assessment system (upper part of Figure 1) is described elsewhere (Cheng and Shen, 2010; Downey et al., 2011). The word error rate (WER) over the test set using the final acoustic models is around $35 \%$.

\subsection{The non-scorable tests}

This research focuses on data obtained from four stages; elementary, primary, middle school and high school. Those consist of 6000 spoken tests (1500 per stage), of which 4800 were used for training (1200 per stage) and the remaining 1200 


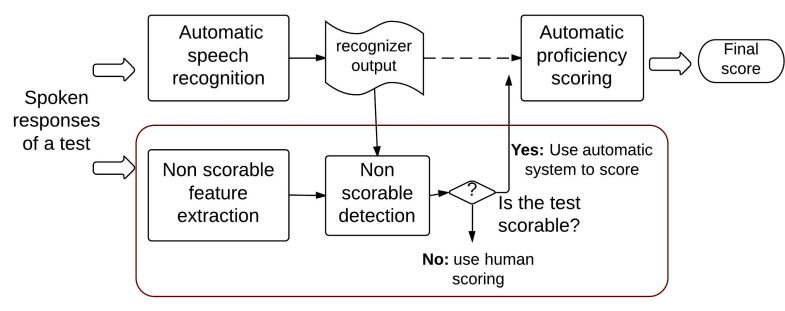

Figure 1: Outline of the assessment system. The lower block is the non-scorable test detection module, that is the focus of this work.

were used for testing. Professional human graders were recruited to provide a grade for each test response, following pre-defined rubrics per item. The grades per test are then summed up to compute an overall human grade in the range 0-14. Each test was double graded and the final human grade was computed by averaging. Our automatic scoring system was also used to estimate an overall machine grade in the range 0-14 for each test, after considering all student responses.

We define a test as non-scorable when the overall machine and human grades differ by more than 3 points. For our dataset of 6000 tests, only 308 (or approx. 5.1\%) are non-scorable, according to this definition. Inspecting a subset of those tests, revealed various reasons that may cause a test to be non-scorable. Those include poor audio quality (recording or background noise, volume too loud or too soft), excessive mouth noises and vocalizations, foreign language, off-topic responses and unintelligible speech (extremely disfluent and mispronounced). As expected, the above issues are more common among younger test takers. Although the cases above can be very different, a commonality is that their ASR results are unreliable, therefore making subsequent automatic scoring inaccurate. In the following sections, we propose new methods for detecting problematic ASR outputs and filtering out non-scorable tests.

\section{Syllable based features}

The intuition behind the syllable based features is to compare information coming from the ASR component with information that is derived directly from the speech signal. If these two sources are inconsistent, this may indicate problems in the recognition output, which often results in nonscorable tests. Here, we focus on syllable locations as the type of information to compare. Syllable locations can be approximated as the vowel lo-
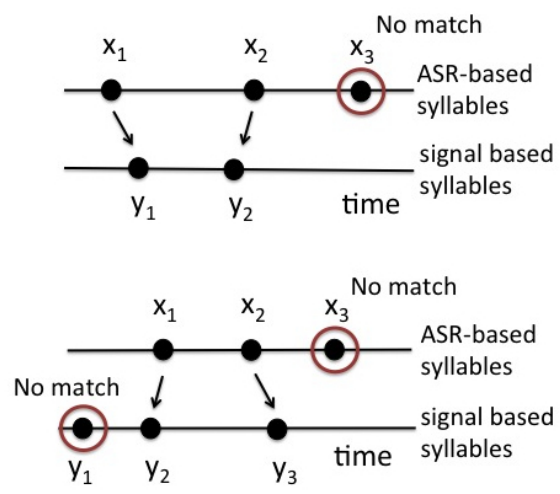

Figure 2: Two examples of mapping between ASR-derived and signal-derived syllable locations.

cations of the speech recognition output. Alternatively, they can be approximated using the speech pitch and intensity signals. By examining intensity, we may find intensity peaks that are preceded by intensity dips, and by examining pitch, we may select voiced intensity peaks as estimates of syllable locations. This method for identifying syllables was described by Jong and Wempe (2009), and the number of syllables has been used as a feature for non-scorable detection in Yoon et al. (2011). In this work, we propose to use the syllable information in order to compute features that measure similarities between signal-derived and ASR-derived syllable locations.

Assume that we have a sequence of $n$ ASRderived syllable locations: $X=\left\{x_{1}, x_{2}, \ldots, x_{n}\right\}$ and a sequence of $m$ signal-derived locations: $Y=\left\{y_{1}, y_{2}, \ldots, y_{m}\right\}$. The first step in computing similarity features is finding a mapping between the two sequences. Specifically, we want to find an appropriate mapping that pairs points $\left(x_{i}, y_{j}\right), x_{i} \in X, y_{j} \in Y$ such that the smallest possible distances $d\left(x_{i}, y_{j}\right)$ are preferred. Potentially inconsistent points can be discarded. Two examples are presented in Figure 2. In the upper example $n>m$, therefore some syllable locations of the longer sequence will be discarded (here location $x_{3}$ ). In the lower example, although $n=m$, the mapping that produces location pairs with the smallest distances is $\left(x_{1}, y_{2}\right)$ and $\left(x_{2}, y_{3}\right)$, while locations $y_{1}, x_{3}$ will be discarded. A mapping $\left(x_{3}, y_{1}\right)$ would be invalid as it violates time constraints, given the existing mappings. We use a greedy algorithm for finding the mapping, which iteratively searches all available valid paired locations and finds the pair $\left(x_{i}, y_{j}\right)$ with the smallest 
distance. A mapping $\left(x_{i}, y_{j}\right)$ is valid if no time constraints are violated, e.g., there is no previously selected mapping $\left(x_{k}, y_{l}\right)$, where $k<i, l>j$ or $k>i, l<j$.

The algorithm is described in Algorithm 1. Our implementation is recursive: after finding the locations that define the best available mapping at each step, the algorithm is recursively called to search for mappings between points that are both either at the right subsequences, or at the left subsequences, with respect to the recent mapping. The right subsequences contain points on the right of the selected mapping (similarly for left subsequences). That way we avoid searching for mappings that would violate the time constraints.

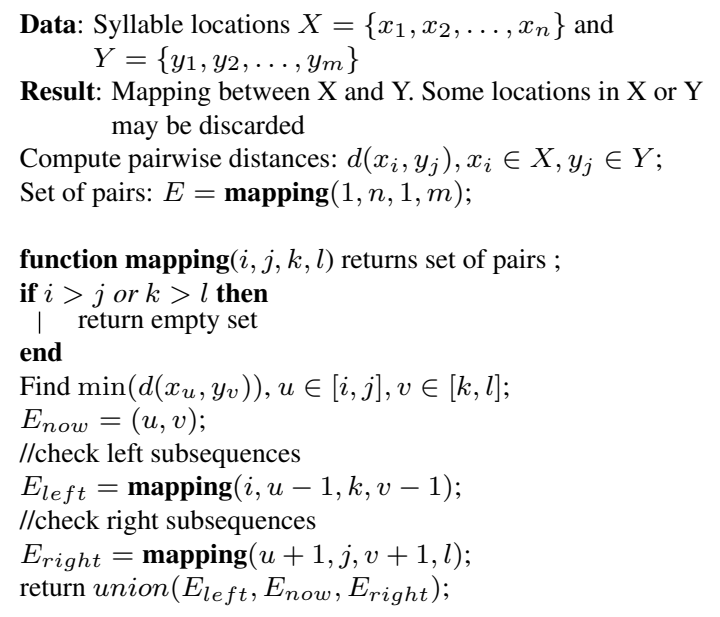

Algorithm 1: Compute mapping between ASRbased and signal-based syllable locations

Based on the mapping of Algorithm 1, we estimate a set similarity features including number of pairs found, number of syllables that were not paired, the absolute length difference between the two sequences, as well as normalized versions of these features (we normalize the features by dividing with the maximum sequence length). For example, in the lower part of Figure 2, there are two pairs and the longest sequence has length three, so the normalized number of pairs found is $2 / 3$. Other features include average, min, max and standard deviation of the distances of the pairs found, as well as the lengths of the two sequences. These features are a set of descriptions of the quality of the mapping or, in other words, of the similarity between the two syllable sequences.

Algorithm 1 follows a greedy approach, however, one could derive a similar mapping using dynamic programming (DP) to minimize the average distance over all selected pairs. In practice, we do

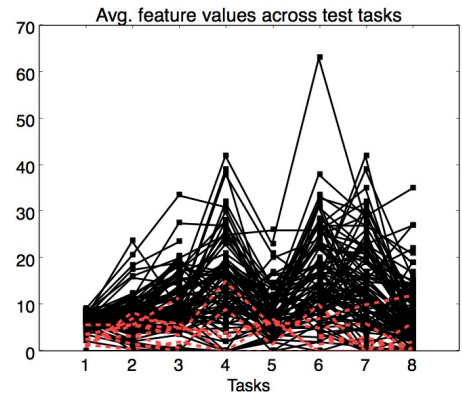

(a) Number of syllable pairs found.

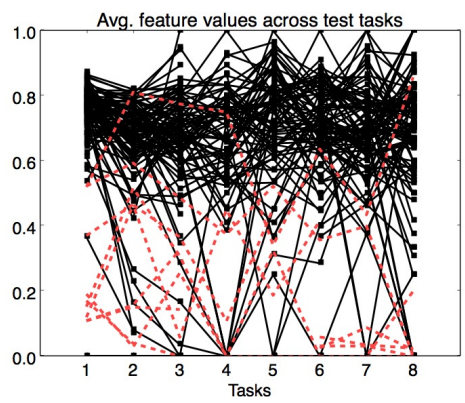

(b) Number of pairs over length of largest sequence.

Figure 3: Visualization of feature values across tasks during a test, for sampled tests. Scorable tests are in black, non-scorable in dashed red lines. For tasks that contain multiple responses, we average the feature values of the responses of a task.

not expect the choice of greedy or DP approach to greatly affect the final computed similarity features, and we chose the greedy approach for simplicity (although DP implementations could be explored in the future).

To visualize the feature information, we plot the feature values across tasks of a test, for randomly sampled tests. For tasks that contain multiple responses (multiple items), we average the feature values of the responses of a task. Figure 3(a) visualizes the number of pairs found. Each test is represented by a set of feature values (one per task) connected by lines between tasks. Values of some tasks may be missing if they are undefined, e.g., the student did not reply. Scorable tests are represented in black, and non-scorable tests in dashed red lines. We notice that the number of pairs found for non-scorable tests is consistently low throughout the tasks of the test. This agrees with our intuition that for non-scorable tests there will be less similarity between the ASR-based and signalbased syllable locations, thus there will be fewer pairs between these two location sequences, compared to scorable tests. Similarly, Figure 3(b) vi- 
sualizes the normalized pairs found, and again this percentage is lower for non-scorable tests, indicating that fewer pairs were found for those tests.

In our implementation, we computed the ASRbased syllable sequences by performing phonemelevel forced alignment of the ASR, and approximating the syllable location as the center of each vowel segment of the force aligned result. We computed the signal-based syllable sequence by augmenting the open source Praat script developed by Jong and Wempe (2009) to output syllable locations. The syllable locations are approximate: computing the syllable detection accuracy would require human annotation of syllables in our corpus, which is out of the scope of this work. Our focus is to estimate syllables well enough, so as to compute useful features. Based on Figures 3(a) and (b) and the results of Section 9, our syllable detection works sufficiently well for our purpose.

\section{Language model based features}

Language models (LMs) are used to model word transition probabilities in ASR systems, and are learnt using large text corpora. For cases where the input speech belongs to a specific topic, it is common to use constrained LMs, e.g., learn the word transitions from corpora related to the topic in question. Here we explore the idea of using different LMs for our ASR system, either highly constrained or unconstrained ones, and comparing the corresponding recognition results. If the ASR results of the two LMs are very different, then it is likely that the ASR result is problematic, which may be indicative of a non-scorable test. To detect those cases, we introduce a set of features that measure the similarity between ASR results obtained using different language models.

In our system, each item requires the user to talk about a specific known topic. The default LM used by our ASR component is item dependent and is constrained on the topic of the item. In general, this is beneficial to our system as it allows the ASR to focus on words that have a high enough likelihood of appearing given the item topic. However, for some non-scorable tests, we noticed that this approach may result in misrecognizing phrases that are off-topic or non-English as valid on-topic phrases. Therefore, we introduce an unconstrained LM to detect cases where the constrained LM causes our system to misrecognize topic specific words that were not actually spoken. We create the



(a) Edit distance over longest sequence length.

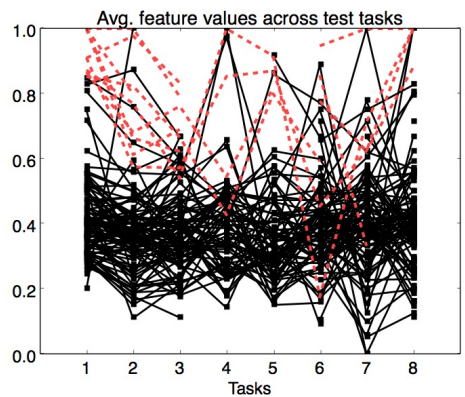

(b) Length difference over longest sequence length.

Figure 4: Visualization of feature values across tasks during a test, for sampled tests. Scorable tests are in black, non-scorable in dashed red lines.

unconstrained LM independent of the vocabulary used, by training a phoneme bigram LM that models phoneme transition probabilities. Hence, our LM can handle out of vocabulary or non-English words that often appear in non-scorable tests.

We use item specific training data to build a standard bigram word LM for each item. For the unconstrained LM, we perform phoneme-level forced alignment of all training data, and build a item independent bigram phoneme LM. We perform recognition using both LMs and compare the resulting phoneme-level recognition results. Comparison is performed by computing the edit distance between the two phoneme sequences, obtained from the two LMs. Edit distance is a common metric for measuring similarity between sequences and estimates the minimum number of insertions, deletions or substitutions required to change one sequence to the other. We compute a number of similarity features including edit distance, length difference between the sequences, number of insertions, deletions and substitutions, as well as normalized versions of those features (by dividing with the maximum sequence length). We also include the two phoneme se- 
quence lengths as features.

Similarly to Section 4, we visualize feature information by plotting feature values across tasks, for randomly sampled tests. The resulting plots for edit distance and length difference between sequences, both normalized, are presented in Figures 4 (a) and (b) respectively. Scorable tests are in black and non-scorable in red dashed lines. Intuitively, the more dissimilar the sequences from the two LMs are, the larger the features values will be for these two features. Looking at the plots, we notice that, as expected, non-scorable tests tend to have larger feature values compared to scorable ones. This indicates that the proposed phoneme LM can help detect cases of non-scorable tests.

\section{Confidence features}

The ASR component of the Pearson assessment system assigns confidence scores to the recognized words. Three variants of confidence scores are computed: mconf (based on normalized acoustic scores), aconf (based on force alignment and phoneme recognition) and lconf (lattice-based). They are described in our previous work (Cheng and Shen, 2011), where they were used for offtopic detection. Here, we use them for nonscorable detection, and compute them separately using the ASR result obtained from either the item specific word LM or the item independent phoneme LM. For each confidence score, our feature set includes the average score value over words of a response, and the maximum, minimum and standard deviation. We also compute the word-level recognition log-likelihood using each of the two LMs, and include as features the average, minimum, maximum and standard deviation of these log-likelihoods over words of a response.

Although the confidence scores are described in Cheng and Shen (2011), here we compute them using the proposed phoneme LM (in addition to the standard word LM), thus they are significantly different from prior work. Indeed, scores computed by the proposed phoneme LM prove to be highly informative (see Section 9, Table 3).

\section{Signal derived and ASR features}

A variety of signal-derived and ASR-based features have been used in the literature for nonscorable detection (Cheng and Shen, 2011; Yoon et al., 2011; Chen and Mostow, 2011), as well as related work on pronunciation and fluency assess- ment (Bernstein et al., 2010; Higgins et al., 2011). In this study, we extract and include a set of common features.

Signal-derived features typically describe properties of the pitch and energy of the speech signal. Our feature set includes maximum and minimum energy, number of nonzero pitch frames and average pitch. We also extract features that estimate noise level, specifically Signal to Noise Ratio (SNR). For SNR estimation we used the NIST Speech Quality Assurance package (NIST, 2009)

Furthermore, we use features extracted from the ASR result, including utterance duration, number of words spoken, number of interword pauses, average interword pause duration, average pause duration before the first spoken word (response latency), and number of hesitations. Pauses, hesitations and response latency have been found informative of speaking fluency (Bernstein et al., 2010), and could be indicative of problematic, non-scorable tests. We also compute two variations of speech rate: words over total response duration and words over duration of speech (excluding pauses). Other ASR features we use include recognition log-likelihood, average LM likelihood, number of phonemes pruned during recognition, and average word lattice confidence. We include some additional confidence-related features, like percentage of low confidence words or phonemes in the response (low confidence is defined based on an experimental threshold).

We compute ASR features that are specific to the task: either repeat or non-repeat. For the repeat tasks, where the student is asked to repeat a prompt sentence, we compute the number of insertions, deletions and substitutions of the recognized response compared to the prompt, as well as the number and percentage of the recognized prompt words. For the open question (non-repeat) tasks, where the student gives an open ended response on a topic, we estimate the number of key words recognized in the response, from a set of predefined, topic key words.

Finally, we also include some features that are not used in previous work, and were devised to enhance earlier versions of our non-scorable detection system. Specifically, we compute the number of clipped energy frames, where clipping happens when energy exceeds a max value (often because the student is speaking too close to the microphone). Also, we include an indicator feature 
that indicates when the number of non zero pitch frames exceeds a certain threshold but the ASR recognizes only silence. This is a rough way to detect inconsistencies between the ASR and the pitch signal, where pitch indicates the presence of voiced speech, but the ASR recognizes silence. Although these features are new, for simplicity, we merge them in our baseline feature set.

Overall, we have extracted a diverse and powerful set of representative features, which will be referred as 'base' feature set, and is summarized in Table 1.

Table 1: Summary of features included in the 'Base' feature set

\begin{tabular}{c|l}
\hline signal & $\begin{array}{l}\text { description } \\
\text { max and min energy, nonzero pitch frames, avg. pitch, num- } \\
\text { ber of clipped frames, SNR }\end{array}$ \\
\hline \multirow{3}{*}{ ASR } & $\begin{array}{l}\text { number of words spoken, pauses and hesitations, utterance } \\
\text { duration, speech rate (2 variations), avg. interword pause du- } \\
\text { ration, leading pause duration. }\end{array}$ \\
\cline { 2 - 2 } & $\begin{array}{l}\text { ASR log-likelihood, average LM likelihood, number of } \\
\text { phonemes pruned, average word lattice confidence, percent- } \\
\text { age of low confidence words and phonemes }\end{array}$ \\
\cline { 2 - 2 } & $\begin{array}{l}\text { Repeat types: number of insertions, deletions, substitutions, } \\
\text { number of recognized prompt words, percentage of recog- } \\
\text { nized prompt words. } \\
\text { Non repeat types: number of recognized key words }\end{array}$ \\
\hline indicator & $\begin{array}{l}\text { indicator when number of zero pitch frames exceeds a thresh- } \\
\text { old while ASR recognizes silence }\end{array}$ \\
\hline
\end{tabular}

\section{Random forest classification}

We use a binary random forest classifier to decide if a test is scorable or not. A random forest is an ensemble of decision trees where each tree decides using a subset of the features and the final decision is computed by combining the tree decisions (Breiman, 2001). Random forests can take advantage of feature combinations to construct a complex, non-linear decision region in the feature space. In addition, they can be trained fast, have good generalization properties and do not require much parameter tuning, which makes them popular classifiers in the machine learning literature. In our work, a variety of diverse reasons may cause a test to be non-scorable, including background or line/static noise, off-topic responses, non-English or unintelligible speech. Random forests combine a number of decision trees that could correspond to the different sub-cases of our problem, therefore they seem well suited for non-scorable test detection. According to our experiments, random forests outperform decision trees and maximum entropy classifiers. Therefore, all results of Section 9 are based on random forest classification.

Up to now, we have described feature extraction for each test response. The non-scorable detection system needs to aggregate multiple response information to make an overall decision at the test level. We can combine response-level features in a straightforward manner by taking their average over a test. However, responses may belong to different types of tasks, either repeat or non repeat ones, and some of the features are task specific. Also, repeat task responses often resemble recited speech, while non-repeat ones tend to be more spontaneous. To preserve this information, we separately average features that belong to repeat responses and non-repeat responses of a test (two averaged features are extracted per test and per feature). There are cases where a feature cannot be extracted for a response, because it is undefined, i.e., for a response that is recognized as silence the average interword pause duration is undefined. Therefore, we also include the percentage of repeat or non-repeat responses used to compute the average, i.e., two percentage features (for repeat and non-repeat cases) are extracted per test and per response. More statistics could be extracted when combining response features, e.g., variance, max and min values, and others. However, our preliminary experiments indicated that including just averages and corresponding percentages is sufficient, and adding more statistics greatly increases the feature vector size without significant performance gains. Therefore, our final feature set includes only averages and percentages.

\section{Experiments and results}

\subsection{Experimental setup}

Our experiments are organized in 5-fold cross validation: we randomly split the 6000 tests into five sets, and each time we use three sets for training the random forest classifier, one set as a development for optimizing the number of trees, and one set for testing non-scorable classification performance. Performance is computed after merging all test set results. Because the percentage of nonscorable tests in our dataset is small (approx. 5\%) and random forests are trained with a degree of randomness, different runs of an experiment can cause small variations in performance. To minimize this effect, we repeat each 5-fold cross validation experiment 10 times, and report the average and standard deviation over the 10 runs.

Performance is estimated using the ROC curve of false acceptance rate (FAR) versus false rejec- 
tion rate (FRR) for the binary (scorable vs nonscorable) classification task. Our goal is to minimize the area under the curve (AUC), e.g., achieve low values for both FAR and FRR. Our experiments were performed using the Python ScikitLearn toolbox (Scikit-Learn, 2014).

\subsection{Results}

Table 2 presents the average AUC performance of non-scorable test detection over 10 experiment runs, using different feature sets and random forests. 'Base' denotes the set of standard ASR-based and signal-based features described in Section 7. Syllable based and LM based denote the similarity features introduced in Sections 4 and 5 respectively. Finally, 'confidence' denotes the confidence and log-likelihood features derived from the standard and the proposed phoneme LM, as described in Section 6. According to our results, 'base' features are the best performing. However, it is encouraging that our proposed comparisonbased syllable and LM approaches, that approach the problem from a different perspective and only use similarity features, still achieve comparable performance.

Table 2: Average and standard deviation of AUC over 10 experiment runs for the different feature sets, and combinations of feature sets.

\begin{tabular}{|c|c|}
\hline features & AUC (Avg \pm Std.dev) \\
\hline \hline Base & $0.102 \pm 0.007$ \\
Syllable based & $0.122 \pm 0.011$ \\
LM based & $0.123 \pm 0.008$ \\
Confidence & $0.106 \pm 0.011$ \\
\hline \multicolumn{2}{|c|}{ Feature Combination } \\
\hline Base+Syllable & $0.091 \pm 0.007$ \\
Base+LM & $0.091 \pm 0.011$ \\
Base+Confidence & $0.094 \pm 0.011$ \\
All & $0.097 \pm 0.011$ \\
\hline Feature Combination (select top 300 features) \\
\hline Base+Syllable & $0.092 \pm 0.008$ \\
Base+LM & $0.088 \pm 0.012$ \\
Base+Confidence & $0.097 \pm 0.010$ \\
All & $0.092 \pm 0.008$ \\
\hline \multicolumn{2}{|c|}{ Classifier Decision Combination } \\
\hline Base+Syllable & $0.087 \pm 0.008$ \\
Base+LM & $0.085 \pm 0.007$ \\
Base+Confidence & $0.084 \pm 0.007$ \\
All & $\mathbf{0 . 0 8 1} \pm \mathbf{0 . 0 0 6}$ \\
\hline
\end{tabular}

Table 2 also presents the AUC performance after concatenating the feature vectors of different feature sets, under 'Feature Combination'. We notice that adding separately each of our proposed syllable based, LM based and confidence features to the base features improves performance by decreasing AUC. This further indicates that the pro-

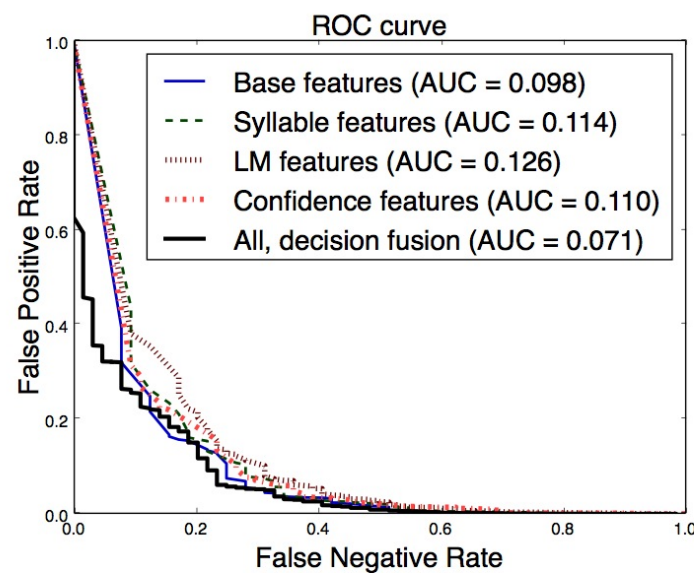

Figure 5: Test set ROC curves for different feature sets, and their combination using decision fusion (averaging), for one run of the experiment.

posed features carry useful information, which is complementary to the 'base' feature set. Combining all features together leads to a relatively small performance increase, possibly because the large number of features may cause overfitting.

We also perform feature selection by selecting the top 300 features from each feature set. Features are ranked based on their positions in the trees of the random forest: features closer to the root of a tree contribute to the decision of a larger number of input samples, thus, the expected fraction of the samples that each feature contributes to, can be used as an estimate of feature importance. We use Scikit-Learn to compute the feature importance for each feature, and rank features based on their average importance over the 10 experiment runs. The results, presented in Table 2, show that feature selection helps for cases of large feature sets, i.e., when combining all features together. However, for cases when fewer features are used, the performance does not change much compared to no feature selection.

Finally, instead of concatenating features, we perform decision combination by averaging the decisions of classifiers trained on different feature sets. For simplicity, we perform simple averaging (in future when a larger train set will be available, we can explore learning appropriate classifier weights, and performing weighted average). From the results of Table 2, we notice that this approach is advantageous and leads to a significant performance increase, especially when we combine all four classifiers: one using existing 'base' features, and the rest using our new features. Overall, we 
Table 3: Top-10 ranked features from each feature set. 'Av' and 'prc' denote that the feature is an average or percentage respectively, while ' $r$ ' and 'nr' denote that the feature is computed over repeat or non-repeat responses, respectively. For the confidence features, 'wLM' denotes the feature is computed using regular bigram word LM and 'pLM' denotes proposed bigram phoneme LM.

\begin{tabular}{c|ll}
\hline feature set & description & \\
\hline \hline \multirow{5}{*}{ signal and ASR } & n_hesitations (av, r) & indicator_pitch_asr (av,r) \\
& min_energy (av,r) & n_pitch_frames (av, nr) \\
& n_pitch_frames (av,r) & asr_loglik (av, nr) \\
& asr_loglik (av,r) & min_energy (av, nr) \\
& avg_pitch(av,nr) & snr (av, nr) \\
\hline \multirow{5}{*}{ syllable based } & diff_lengths_norm (av,r) & diff_lengths_norm (av,nr) \\
& min_pair_distances(av,nr) & diff_lengths (av,r) \\
& n_pairs_norm (av,nr) & diff_lengths(av,nr) \\
& avg_pair_distances (av,r) & min_pair_distances (av,r) \\
& n_pairs_norm (av,r) & max_pair_distances (av,nr) \\
\hline \multirow{5}{*}{ LM based } & edit_dist_norm (av,r) & diff_lengths_norm (av,r) \\
& n_insert_norm (av,r) & edit_dist_norm (av,nr) \\
& diff_lengths_norm (av, nr) & n_insert_norm (av,nr) \\
& n_substitute_norm (av,nr) & min_length (av,nr) \\
& min_length (av,r) & n_substitute (av, nr) \\
\hline \multirow{5}{*}{ Confidence } & avg_aconf_pLM (av,nr) & min_loglik_pLM (av,r) \\
& min_loglik_pLM (av,nr) & max_lconf_pLM (av,r) \\
& min_aconf_pLM (av,nr) & stddev_loglik_pLM (av,nr) \\
& min_loglik_wLM (av,r) & min_aconf_pLM (av,r) \\
& std_loglik_pLM (av,r) & avg_loglik_pLM (av,r) \\
\hline
\end{tabular}

achieved a decrease in AUC from 0.102 to 0.081, a $21 \%$ relative performance improvement.

Figure 5 presents the ROC curves for one run of the experiment, for the four feature sets, and their combination using averaging of the classifier decisions. Combining all feature sets leads to a lower AUC (thick black line). We notice improvement especially in reducing false positives, e.g., misclassifying scorable test as non-scorable.

In Table 3, we present the top 10 selected features from each feature set, based on their averaged feature importance. Overall, we notice that both repeat and non-repeat features are among the top ranked, indicating that both types are informative. Only average features are among the top ranked, which suggests that averages carry more information than percentage features. For the syllable and LM features, we can see many intuitive similarity features being at the top, such as difference of sequence lengths, edit distance and number of insertions (LM based feature set), and average, min and max of the distances of paired syllables (syllable based feature set). For confidence, we note that many log-likelihood features are at the top (here log-likelihood statistics are computed over words of a response). Also, note that the great majority of top-ranked confidence features are computed using our proposed item independent phoneme LM, instead of the regular item de- pendent word LM, indicating the usefulness of this approach.

\section{Conclusion and future work}

In this work, we have proposed new methods for detecting non-scorable tests in spoken language proficiency assessment applications. Our methods compare information extracted from different sources when processing a test, and compute similarity features. Inconsistencies suggest problematic ASR, which is often indicative of nonscorable tests. We extract two sets of features: syllable based, which compare syllable location information, and LM based, which compare ASR obtained using item specific and item independent LMs. Our proposed item independent LM is a bigram phoneme LM, which can handle out-ofvocabulary or non-English words, that often appear in non-scorable tests. By visualizing the proposed similarity features, we verify that they can highlight inconsistencies that are common in nonscorable tests. We experimentally validate our methods in a large, challenging dataset of young ELLs interacting with the Pearson spoken assessment system. Our features carry complementary information to existing features, and when combined with existing work, they achieve a $21 \%$ relative performance improvement. Our final, nonscorable detection system combines the decisions of four random forest classifiers: one using baseline features, and the rest using proposed features.

We are currently collecting human annotations for non-scorable tests in our dataset, which contain additional annotation of the different non-scorable subcases in these tests, e.g., noise, off-topic, nonEnglish, unintelligible speech etc. In the future, we plan to use these annotations to further validate our methods, as well as perform detailed evaluation of the usefulness of our proposed feature sets for each of the non-scorable test subcases.

\section{References}

J. Bernstein and J. Cheng. 2007. Logic and validation of a fully automatic spoken English test. In V. M. Holland and F. P. Fisher, editors, The Path of Speech Technologies in Computer Assisted Language Learning, pages 174-194. Routledge, New York.

J. Bernstein, J. De Jong, D. Pisoni, and B. Townshend. 2000. Two experiments on automatic scoring of spoken language proficiency. In Proc. of STIL (Integrating Speech Technology in Learning). 
J. Bernstein, A. Van Moere, and J. Cheng. 2010. Validating automated speaking tests. Language Testing, 27.

L. Breiman. 2001. Random forests. Machine Learning, 45 .

W. Chen and J. Mostow. 2011. A tale of two tasks: Detecting children's off-task speech in a reading tutor. In Proc. of Interspeech.

J. Cheng and J. Shen. 2010. Towards accurate recognition for children's oral reading fluency. In Proc. of IEEE-SLT, pages 91-96.

J. Cheng and J. Shen. 2011. Off-topic detection in automated speech assessment applications. In Proc. of Interspeech.

J. Cheng, J. Bernstein, U. Pado, and M. Suzuki. 2009. Automated assessment of spoken modern standard arabic. In Proc. of the Fourth Workshop on Innovative Use of NLP for Building Educational Applications.

R. Downey, D. Rubin, J. Cheng, and J. Bernstein. 2011. Performance of automated scoring for children's oral reading. In Proc. of the 6th Workshop on Innovative Use of NLP for Building Educational Applications.

M. Eskanazi. 2009. An overview of spoken language technology for education. Speech Communication, 51 .

D. Higgins, X. Xi, K. Zechner, and D. Williamson. 2011. A three-stage approach to the automated scoring of spontaneous spoken responses. Computer Speech and Language, 25.

N. H. De Jong and T. Wempe. 2009. Praat script to detect syllable nuclei and measure speech rate automatically. Behavior research methods, 41:385-390.

NIST. 2009. The NIST SPeech Quality Assurance (SPQA) Package. http://www.nist.gov/ speech/tools/index.htm.

G. Pearson. 2006. Ask NCELA No.1: How many school-aged English-language learners (ELLs) are there in the U.S.? Washington, D.C: National Clearing House for English-Language Acquisition and Language Instruction Educational Programs 2006, Retrieved Online February 2007 at http://www.ncela.gwu.edu/expert/ faq/01leps.htm.

Pearson. 2011. Skills and scoring in PTE Academic. http://www.pearsonpte.com/ SitecollectionDocuments/US_Skills_ Scoring_PTEA_V3.pdf.

Scikit-Learn. 2014. The Scikit-Learn Machine Learning Python Toolbox. http://scikit-learn. org/.
J. Tepperman, M. Black, P. Price, S. Lee, A. Kazemzadeh, M. Gerosa, M. Heritage, A. Alwan, and S. Narayanan. 2007. A Bayesian network classifier for word-level reading assessment. In Proc. of Interspeech.

X. Xu, M. Suzuki, and J. Cheng. 2012. An automated assessment of spoken Chinese: Technical definition of hanyu standards for content and scoring development. In Proc. of the Seventh International Conference \& Workshops on Technology \& Chinese Language Teaching.

S.-Y. Yoon, K. Evanini, and K. Zechner. 2011. Nonscorable response detection for automated speaking proficiency assessment. In Proc. of the Sixth Workshop on Innovative Use of NLP for Building Educational Applications. 